Wie sieht es mit der heimischen Versorgung aus?

I.Strillinger: Das Problem ist die Dauer bis zur Diagnose. In der Zeit davor sind die Betroffenen oftmals frustriert, da sie häufig nicht ernst genommen werden und ihre Symptome als Einbildung abgetan werden.

Auch bei einer Untersuchung beim Augenarzt könnte Morbus Fabry festgestellt werden. Sie können Ablagerungen in den Augen erkennen und dadurch auf Morbus Fabry schließen.
Wurde bereits die Diagnose gestellt, dann ist die Versorgung gut. Der Weg dahin ist das Problem. Wünschenswert wäre eine Sensibilisierung für diese Erkrankung.

\section{Herzlichen Dank für das Gespräch!}

Das Interview führte Dr. Philip Klepeisz

\section{Morbus Fabry Selbsthilfegruppe}

Weitere Informationen und Kontaktda ten heimischer Morbus Fabry Zentren: Iris Strillinger, Obfrau des Morbus Fabry Selbsthilfevereins Österreich Tel.: +436641536251 E-Mail: info@morbus-fabry.eu www.morbus-fabry.eu

psychopraxis.neuropraxis2018.21:204-205 https://doi.org/10.1007/s00739-018-0526-7 (c) Springer-Verlag GmbH Austria, ein Teil von Springer Nature 2018

\title{
ASS-Dosis: Abhängig vom Körpergewicht
}

Neue Studiendaten legen nahe, dass niedrig dosierte Acetylsalicylsäure (ASS) ab einem Körpergewicht von 70 kg nicht ausreichend vor Schlaganfall, Herzinfarkt oder anderen vaskulären Ereignissen schützt.

Die Wirksamkeit von Acetylsalicylsäure (ASS) zur Vorbeugung von Herzinfarkten und Schlaganfällen hängt stark vom Körpergewicht ab. Eine Publikation im renommierten Fachmagazin „The Lancet" lässt vermuten, dass lediglich ein Bruchteil der Risikopatienten mit der Standarddosis von 75 bis 100 Milligramm ASS ausreichend geschützt ist. Ab einem Körpergewicht von 70 Kilogramm, so zeigte die Analyse von 14 Studien, sind niedrige ASS-Dosen weniger wirksam. Gleichzeitig scheinen leichtere Menschen nicht von höheren Dosen zu profitieren. Die Deutsche Gesellschaft für Neurologie (DGN) und die Deutsche Schlaganfall-Gesellschaft (DSG) appellieren vor diesem Hintergrund für neue Studien mit dem alten Medikament. Die derzeit praktizierte "One Dose Fits All“-Strategie muss daher laut Experten neu bewertet werden.

\section{Standarddosis wirkt nur bei Leicht- gewichten}

Exakt 117.279 Menschen hatten an diesen Studien teilgenommen. Sie wurden anhand des Körpergewichts in
Schritten von je zehn Kilogramm und anhand der Körpergröße in Schritten von je zehn Zentimetern in verschiedene Gruppen eingeteilt. Dabei stellte sich heraus, dass niedrig dosiertes ASS (75-100 Milligramm täglich) bei Menschen zwischen 50 und 69 Kilogramm das Risiko für ein kardiovaskuläres Ereignis um durchschnittlich $25 \%$ reduzierte. Schon ab 70 Kilogramm zeigte sich aber kein eindeutiger Nutzen mehr, und die Sterblichkeit bei einem ersten Ereignis war für Personen ab 70 Kilogramm sogar um ein Drittel erhöht.

Umgekehrt war hoch dosiertes ASS ( $\geq 325$ Milligramm täglich) nur bei relativ schweren Menschen ab 70 Kilogramm geeignet, um Herzinfarkte und Schlaganfälle zu verhindern, nicht aber bei denjenigen mit einem Gewicht unterhalb der 70-Kilogramm-Schwelle.

\section{Vor allem Männer nicht ausrei- chend geschützt}

Die Analysen von Rothwell und Mitarbeitern belegen überzeugend, dass das Körpergewicht einen erheblichen Einfluss auf die optimale Dosis in der
Primär- und Sekundärprophylaxe des Schlaganfalls besitzt. Etwa $80 \%$ aller Männer und die Hälfte aller Frauen wiegen mehr als 70 Kilogramm. Es kann davon ausgegangen werden, dass sehr viele Menschen in der Primär- und Sekundärprophylaxe unterversorgt sind. Die Gruppe der Neurologen halten vertiefende Forschungen für dringend notwendig - auch im Hinblick auf eine eventuelle Neubewertung der derzeit gültigen Leitlinienempfehlungen. „Wünschenswert wäre eine randomisierte Studie in der Sekundärprävention nach transienter ischämischer Attacke und ischämischem Insult bei Personen mit einem Körpergewicht von über 70 Kilogramm", so die Experten.

\section{Literatur \\ 1. Rothwell PM et al (2018) Effects of aspirin on risks of vascular events and cancer according to bodyweight and dose: analysis of individual patient data from randomised trials. Lancet. S0140-6736 (18) 31133-31134}

psychopraxis. neuropraxis 2018 $\cdot 21: 205$ https://doi.org/10.1007/s00739-018-0524-9 (c) Springer-Verlag GmbH Austria, ein Teil von Springer Nature 2018 